\title{
Héctor Wittwer (2020) Das Leben beenden. Über eine Ethik der Selbsttötung
}

\author{
Mentis Verlag, Paderborn, 281 Seiten, 34,90€, \\ ISBN 978-3-95743-138-7
}

\section{Urban Wiesing}

Online publiziert: 10. März 2020

(C) Der/die Autor(en) 2020

Der Streit um die Sterbehilfe ist in die Jahre gekommen, in der Bundesrepublik hoch emotionalisiert, die Argumente liegen auf dem Tisch, sind lange bekannt. Die Gräben der Gegner und Befürworter einer Liberalisierung sind verfestigt, die Diskussion ist verfahren. Was kann eine weitere Publikation da noch beitragen?

Ganz einfach: sachliche Gründe und präzise Argumentationen. Und die liefert Héctor Wittwer reichlich und überzeugend, mit breitem Rückgriff auf die philosophische Tradition, und nicht zuletzt, indem er wichtige Differenzierungen beachtet. Die Frage, ob der Suizid an sich moralisch gut sei oder nicht, werde der Komplexität der Situationen nicht gerecht. Man müsse fragen, wie ein Suizid in welchen Situationen zu bewerten sei. Wittwer untersucht zahlreiche Einwände gegen die Möglichkeit eines moralisch zulässigen Suizids. Das Ergebnis: „Es gibt keine überzeugende Begründung für ein ausnahmsloses moralisches Verbot der Selbsttötung.“ (S. 197) Denn schließlich sei das Ursprungsmotiv für seriöse Überlegungen zur Sterbehilfe höchst gewichtig, ,das moralische Recht auf Selbstbestimmung“ (S. 197). Allerdings fände dieses Recht seine Grenzen, wenn Entscheidungen für einen Suizid unvernünftig getroffen würden. Dann - wohl in der Mehrheit der Fälle - ließe sich ein paternalistisches Recht begründen, vor den Folgen der Tat zu bewahren. Aber eben nicht in allen Fällen. Und dort gebe es keine hinreichenden Argumente, warum unter bestimmten Bedingungen die Beihilfe dazu nicht gerechtfertigt sein sollte.

Der Autor plädiert für eine Freigabe des ärztlich assistierten Suizids bei Volljährigen, die an einer unheilbaren körperlichen Krankheit leiden, nach Urteil von Experten zurechnungsfähig sind und über Alternativen aufgeklärt wurden (S. 221). Er wendet sich gegen die Zulassung einer Tötung auf Verlangen.

Prof. Dr. U. Wiesing $(\bowtie)$

Tübingen, Deutschland

E-Mail: urban.wiesing@uni-tuebingen.de 
Einige Bemerkungen vom Rezensenten: Die philosophische Auseinandersetzung mit der Tötung auf Verlangen - Wittwer spricht zumeist von der direkten aktiven Sterbehilfe - bleibt vergleichsweise kurz. Stattdessen werden pragmatische Argumente angeführt, insbesondere die Irrtumswahrscheinlichkeit angesichts der Irreversibilität der Folgen. Wittwer führt zudem die Entwicklung in den Niederlanden an. Dort habe sich eine Kultur des Sterbens etabliert, die das Ursprungsmotiv, die Selbstbestimmung des Patienten, in vielen Fällen aus den Augen verloren habe. Hierbei greift Wittwer aber auf die Sekundärliteratur zurück, die klar von den Gegnern der niederländischen Praxis geschrieben ist. Man hätte sich gewünscht, die Argumente der Befürworter dieser Praxis zu hören, auch wenn man sie nicht unbedingt teilen muss.

Bei der Kritik an der Ausweitung der Sterbehilfe sollte man überdies differenzieren: Die Ausweitung der Tötung auf Verlangen auf Kinder ist ganz anders zu bewerten als die Ausweitung auf psychisch Kranke. Der erste Fall hat mit nichts mehr mit dem Ursprungsmotiv zu tun, da kein rationales Verlangen oder überhaupt kein Verlangen bei (Klein)kindern vorausgesetzt werden kann. Wittwers Kritik an dieser Ausweitung ist berechtigt. Die Ausweitung auf psychische Erkrankungen, die mit erheblichem Leid für die Patienten einhergehen, bleibt hingegen in der Ursprungsargumentation. Hier wäre zu klären, inwieweit sie behandelbar sind im Gegensatz zu manch onkologischer oder neurodegenerativer Erkrankung. Aber diese „Ausweitung“ bleibt in der Argumentation der Selbstbestimmung. Kurzum: Die "Ausweitungen" in den Niederlanden und Belgien sind unterschiedlichen Argumenten zugänglich - oder eben nicht!

Wittwer geht davon aus, dass es bei einer ausschließlichen Zulassung der Beihilfe zum Suizid „zu wenigen tragischen Fällen“ (S. 256) kommen werde, bei denen sich Menschen selbst töten wollen, es aber aufgrund z. B. fehlender motorischer Fähigkeiten nicht können. Hier wäre zu ergänzen, dass moderne Maschinen es auch diesen Menschen erlauben, sich selbst zu töten. Daraus lässt sich dann auch schließen: Man braucht die Tötung auf Verlangen nicht.

Man könnte über die von Wittwer vorgeschlagene Begrenzung der Beihilfe zum Suizid auf körperliche unheilbare Leiden streiten, will die Medizin doch die Trennung somatisch/psychisch gerne überwinden. Das sind freilich Details, die sich in einer komplexen Fragestellung nur begrenzt politisch lösen lassen. Hier wird man um einen gewissen pragmatischen Dezisionismus auf regulatorischer Ebene nicht umhinkommen.

Gegen Ende verweist Wittwer auf eine grundlegende argumentative Asymmetrie. Die Liberalen wollten niemanden vorschreiben, wie sie sterben sollen, sondern Regelungen schaffen, die es jedem erlauben, nach eigenen Maßstäben zu entscheiden. Kein Christ, überhaupt: niemand solle dazu gedrängt werden, Beihilfe zum Suizid in Anspruch zu nehmen. Viele Christen hingegen - darunter zahlreiche kirchliche Vertreter - wollten ihre Vorstellung für alle verbindlich machen.

Héctor Wittwer reiht sich in den Chor derer ein, die den § 217 für misslungen halten. Das Bundesverfassungsgericht hat dies inzwischen mit aller Deutlichkeit bestätigt. Doch nun stellt sich die Frage, wie die Beihilfe zum Suizid geregelt werden soll. Auch wenn es bislang nicht danach aussah: Mögen sich die Verantwortlichen in Politik und Ärzteschaft bei einer Neuregelung die Klarheit, Präzision, Nüchternheit 
und Rationalität zum Maßstab nehmen, die dieses Buch auszeichnet. Ohnehin sei das Buch allen zur Lektüre empfohlen, denen an einer sachlichen Diskussion um die Sterbehilfe gelegen ist.

Funding Open Access funding provided by Projekt DEAL.

Open Access Dieser Artikel wird unter der Creative Commons Namensnennung 4.0 International Lizenz veröffentlicht, welche die Nutzung, Vervielfältigung, Bearbeitung, Verbreitung und Wiedergabe in jeglichem Medium und Format erlaubt, sofern Sie den/die ursprünglichen Autor(en) und die Quelle ordnungsgemäß nennen, einen Link zur Creative Commons Lizenz beifügen und angeben, ob Änderungen vorgenommen wurden.

Die in diesem Artikel enthaltenen Bilder und sonstiges Drittmaterial unterliegen ebenfalls der genannten Creative Commons Lizenz, sofern sich aus der Abbildungslegende nichts anderes ergibt. Sofern das betreffende Material nicht unter der genannten Creative Commons Lizenz steht und die betreffende Handlung nicht nach gesetzlichen Vorschriften erlaubt ist, ist für die oben aufgeführten Weiterverwendungen des Materials die Einwilligung des jeweiligen Rechteinhabers einzuholen.

Weitere Details zur Lizenz entnehmen Sie bitte der Lizenzinformation auf http://creativecommons.org/ licenses/by/4.0/deed.de. 